\title{
Hyperfine structure in pionic atoms: relativistic calculation
}

\author{
M. Trassinelli and P. Indelicato \\ Laboratoire Kastler Brossel, \\ École Normale Supérieure et Université Pierre et Marie Curie, \\ Case 74, 4 Place Jussieu, F-75005 Paris, France
}

\begin{abstract}
We present the hyperfine structure (HFS) energies calculation for pionic atoms. These systems are formed of an atomic nucleus and of negatively charged pion (a spin-0 particle) replacing the electrons. The evaluation of the HFS sub-level energies are essential to estimate the broadening and mean value shift in the experimental atomic lines due to the HFS. The formulas are developed in a completely relativistic framework, using the multipole expansion of the nuclear potential in the Klein-Gordon equation.
\end{abstract}

\section{Introduction}

We present the calculation of the hyperfine structure in pionic atoms, i.e., atoms formed by an ordinary nucleus and a charged pion, instead of replacing the ordinary electrons. The pion is a boson of spin zero and the system is well described by the Klein-Gordon equation $(\mathrm{KG})$ in a central Coulomb potential generated by the atomic nucleus. The interaction between the nuclear magnetic moment and the orbital magnetic moment of the pion create a level splitting. This splitting can be calculated from the multipole expansion of the nuclear electromagnetic potential $[9,4]$ and from the Klein-Gordon equation.

In the next section, we develop the formalism to calculate the energy correction due to the hyperfine interaction term.

In Sec. 3, we will calculate the hyperfine structure correction for a particular case: the pionic nitrogen atom. This system is of particular interest because it is used in an ongoing experiment at the Paul Scherrer Institute to obtain a measurement of the pion mass [10]. 


\section{Hyperfine structure calculation for the Klein-Gordon equation}

The dynamic of a spin-zero particle in an electromagnetic field is described by the KleinGordon equation. For a negatively charged boson (with a charge equal to the electron one) in presence of a central electromagnetic potential, the KG equation can be written as:

$$
\left(\frac{1}{c^{2}}(E-e V(r))^{2}+\frac{\hbar^{2}}{r} \frac{\partial^{2}}{\partial r^{2}} r-\hbar^{2} \frac{l(l+1)}{r^{2}}-\mu^{2} c^{2}-\hat{W}(\mathbf{r})\right) \psi_{n l}(r)=0,
$$

where $\mu$ is the reduced mass of the system, $V(r)$ is the Coulomb potential of the nucleus, $\psi_{n l}(r)$ is the wave-function radial part and $\hat{W}(\mathbf{r})$ is the perturbation term due to the magnetic interaction with the nucleus. The perturbation term can be expressed as:

$$
\hat{W}(\mathbf{r})=-i e \hbar\left(\partial_{i} A^{i}(\mathbf{r})+A_{i}(\mathbf{r}) \partial^{i}\right) .
$$

In Eq. (2) the quadratic term $e^{2} A^{i} A_{i}$ can be omitted in this case as it does not contribute to the HFS splitting. The energy correction due to $\hat{W}$ can be calculated with a linearization of the KG equation obtained by a simple variable change. Once we have a linear equation, it is possible to calculate the correction in first order to the Coulomb energy using standard techniques: $E=E_{(0)}+E_{(1)}$, where $E_{(0)}=\mu c^{2}+\mathcal{E}_{0}^{n l}$ is the total energy of the system without perturbation (mass energy plus binding energy of the atom) and $E_{(1)}$ is the first order correction due to $\hat{W}$,

$$
E_{(1)}^{n l}=\frac{c^{2}\langle W\rangle}{E_{(0)}^{n l}\left(1+\frac{\mu^{2} c^{4}}{\left(E_{(0)}^{n l}\right)^{2}}\right)} .
$$

The expression for $W(\vec{r})$ can be derived by employing the standard multipole expansion of the vector potential $\mathbf{A}(\mathbf{r})$ using spherical tensors $[9,4]$.

$$
\mathbf{A}(\mathbf{r})=-i \frac{\mu_{0}}{4 \pi} \sum_{k}\left(\frac{k+1}{k}\right)^{1 / 2} r^{-k-1} \mathbf{C}^{k k} \circ \hat{\mathbf{M}}^{k}
$$

Here, the symbol o indicates the scalar product between spherical tensor operators $U^{k} \circ$ $V^{k}=\sum_{q}(-1)^{q} U_{q}^{k} V_{-q}^{k}, \hat{\mathbf{M}}^{k}$ is the spherical tensor operator for the nuclear part of the wave function and $\mathbf{C}^{k k}$ is the vector spherical harmonic. If we limit ourselves to the first magnetic multipole contribution we have:

$$
\hat{W}(\mathbf{r})=-e \mu_{0} \hbar \frac{\sqrt{2}}{2 \pi} r^{-2}\left(\mathbf{C}^{11} \cdot \vec{\nabla}\right) \circ \hat{\mathbf{M}}^{1}
$$

where $\hat{\mathbf{M}}^{1}$ is the nuclear magnetic moment tensor.

Using the properties of the spherical tensors and of the vector spherical harmonics $[8,5]$ 
we can finally find the expression for the hyperfine energy correction:

$$
E_{(1)}^{n l F}=\frac{\mu_{I} \mu_{N} e \mu_{0} \hbar}{2 \pi \mu\left(1+\frac{\mathcal{E}_{0}^{n l}}{\mu c^{2}}\right)\left[1+\left(1+\frac{\mathcal{E}_{0}^{n l}}{\mu c^{2}}\right)^{-2}\right]} \frac{F(F+1)-I(I+1)-l(l+1)}{2 I}\left\langle n l\left|\hat{r}^{-3}\right| n l\right\rangle,
$$

where $\mu_{I}$ is the nuclear dipole momentum in units of nuclear magneton $\mu_{N}=(e \hbar) /\left(2 m_{p} c\right)$.

It is interesting to compare this result to the hyperfine energy correction calculated using the non-relativistic Schrödinger equation. In this case we have [3]

$$
\begin{aligned}
E_{(1)}^{N R}=\mu_{I} \mu_{N} \frac{e \mu_{0} \hbar}{4 \pi \mu} \frac{F(F+1)-}{} \frac{I(I+1)-l(l+1)}{2 I}\left\langle n^{\prime} l\left|\hat{r}^{-3}\right| n l\right\rangle_{N R} \\
=\mu_{I} \mu_{N} \frac{e c^{3}(Z \alpha)^{3}}{n^{3}} \mu^{2} \frac{F(F+1)-I(I+1)-l(l+1)}{I(l+1)(2 l+1) l} .
\end{aligned}
$$

The equivalent formula for the $\mathrm{KG}$ equation can be obtained using an expansion in $Z \alpha$ of the relativistic wave-function and energy [7]

$$
\begin{aligned}
& \psi_{n l}(\rho)=\psi_{n l}(\rho)_{N R}\left(1+\left(\frac{25}{36}-\frac{\gamma}{3}-\frac{\ln (\rho)}{3}\right)(Z \alpha)^{2}+\mathcal{O}\left((Z \alpha)^{4}\right)\right) \\
& \mathcal{E}_{0}^{n l}=-\mu \frac{(Z \alpha)^{2}}{2 n}-\mu \frac{(Z \alpha)^{4}}{n^{3}}\left(\frac{1}{2(l+1 / 2)}-\frac{3}{8 n}\right)+\mathcal{O}\left((Z \alpha)^{6}\right) .
\end{aligned}
$$

In particular we have

$$
\left\langle n l\left|\hat{r}^{-3}\right| n l\right\rangle=\left\langle n l\left|\hat{r}^{-3}\right| n l\right\rangle_{N R}\left(1+\mathcal{O}\left((Z \alpha)^{2}\right)\right) .
$$

Using these developments it easy to show that

$$
E_{(1)}^{N R}=E_{(1)}\left(1+\mathcal{O}\left((Z \alpha)^{2}\right)\right)
$$

When we study the non-relativistic limit $c \rightarrow \infty$, we can drop the terms of order $\mathcal{O}\left((Z \alpha)^{2}\right.$ and as expected, Eq. (6) reproduces the non-relativistic expression of Eq. (7).

\section{Hyperfine structure for $n=5 \rightarrow n=4$ transitions for pionic nitrogen}

In this section, we present a specific numerical application of the formalism developed in Sec. 2 for the hyperfine sub-level in pionic nitrogen for the $n=5 \rightarrow n=4$ transitions. Because of the differences in transition probabilities between the (unresolved) hyperfine sub-levels, the hyperfine interaction can produce a shift of the mean energy value of the observed transition. The transition probabilities has been evaluated using the nonrelativistic formula $[3,2]$ assuming a statistical population of the HFS sub-levels (i.e., proportional to $2 F+1$ ). The results are summarized in Table 1 and in Fig. 1 . We notice that, with the statistical population hypothesis, all the transition with a $n s$ final state 
Table 1: Hyperfine transitions energy and transition rate (preliminary results).

\begin{tabular}{ccrrr}
\hline Transition & F-F' & Trans. rate $\left(\mathrm{s}^{-1}\right)$ & Trans. E $(\mathrm{eV})$ & Shift $(\mathrm{eV})$ \\
\hline $5 f \rightarrow 4 d$ & $4-3$ & $4.57 \cdot 10^{13}$ & 4057.68762 & 0.0061 \\
& $3-2$ & $3.16 \cdot 10^{13}$ & 4057.69708 & -0.0034 \\
& $3-3$ & $2.98 \cdot 10^{13}$ & 4057.68457 & 0.0091 \\
& $2-1$ & $2.13 \cdot 10^{13}$ & 4057.70313 & -0.0095 \\
& $2-2$ & $2.25 \cdot 10^{13}$ & 4057.69479 & -0.0011 \\
$5 y \rightarrow 4 d$ & $2-3$ & $1.13 \cdot 10^{11}$ & 4057.68229 & 0.0114 \\
& $5-4$ & $7.13 \cdot 10^{13}$ & 4055.37793 & 0.0030 \\
& $4-3$ & $5.47 \cdot 10^{13}$ & 4055.38210 & -0.0011 \\
& $4-4$ & $5.27 \cdot 10^{13}$ & 4055.37616 & 0.0048 \\
& $3-2$ & $4.17 \cdot 10^{13}$ & 4055.38514 & -0.0042 \\
& $3-3$ & $3.65 \cdot 10^{12}$ & 4055.38068 & 0.0003 \\
& $3-4$ & $5.79 \cdot 10^{10}$ & 4055.37474 & 0.0062 \\
\hline
\end{tabular}

Hyperfine structure for $5 f-4 d$ transition for piN

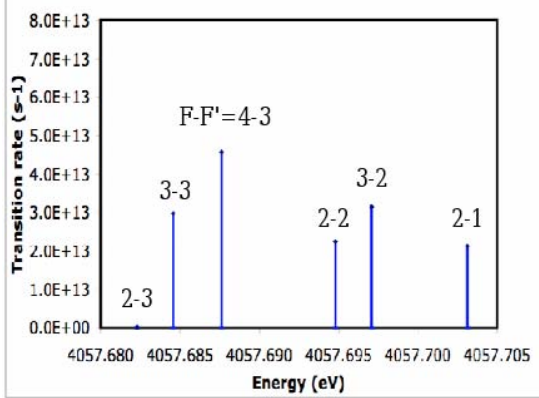

Hyperfine structure for $\mathbf{5 g - 4 f}$ transition for piN

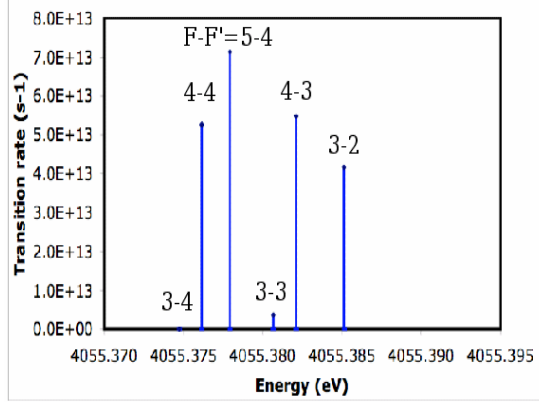

Figure 1: Hyperfine sub-level transition rates for $5 f \rightarrow 4 d$ levels (left) and $5 g \rightarrow 4 f$ levels (right).

are not subject to any HFS shift because the absence of sub-levels for $s$ states. For the transitions between circular levels used for the determination of the pion mass from pionic nitrogen, there is a shift of 0.27 and $0.85 \mathrm{meV}$ respectively, for the $5 g \rightarrow 4 f$ and $5 f \rightarrow 4 d$ transition mean values. These values correspond to a relative corrections of 0.06 and 0.21 ppm respectively to the transition energies. This has to be compared with the $\approx 1.4 \mathrm{ppm}$ accuracy expected for the pion mass energy.

\section{Conclusions}

In the previous section we demonstrate the possibility to calculate the hyperfine structure perturbation terms for the Klein-Gordon equation. The validity of this method is 
confirmed by the non-relativistic limit of the hyperfine energy correction (Eq. (6)) which reproduce the Schrödinger equation results when $c \rightarrow \infty$. Moreover, this method has the advantage to consider the relativistic corrections in a simple way, without introduction to additional operators like in Breit-Pauli Hamiltonian methods $[1,6]$.

\section{Acknowledgments}

Laboratoire Kastler Brossel is Unité Mixte de Recherche du CNRS n 8552.

\section{References}

[1] W. A. Barker and F. N. Glover. Reduction of Relativistic Two-Particle Wave Equations to Approximate Forms. III. Phys. Rev., 99(1):317-324, 1955.

[2] V. Béretetski, E. Lifchitz, and L. Pitayevsky. Électrodynamique Quantique. Physique Theorique. Éditions MIR, Moscou, second edition, 1989.

[3] H. B. Bethe and E. E. Salpeter. Quantum mechanics of one- and two-electron atoms. Springer-Verlag, first edition, 1957.

[4] K.T. Cheng and W.J. Childs. Ab initio calculation of $4 f^{N} 6 s^{2}$ hyperfine structure in neutral rare-earth atoms. Phys. Rev. A, 31(5):2775-2784, 1985.

[5] A. R. Edmonds. Angular Momentum in Quantum Mechanics. Princeton University Press, third edition, 1974.

[6] Michael I. Eides, Howard Grotch, and Valery A. Shelyuto. Theory of Light Hydrogenlike Atoms. Physics Reports, 342(2-3):63-261, 2001.

[7] T. Ericson and W. Weise. Pions and Nuclei, volume 74 of International Series to Monographs on Physics. Oxford Science Publications, 1988.

[8] I. Lindgren and J. Morrison. Atomic Many-Body Theory. Atoms and Plasmas. Springer, Berlin, second edition, 1982.

[9] I. Lindgren and A. Rosén. Relativistic self-consistent-field calculations with application to atomic hyperfine interaction. Case Studies in Atomic Physics, 4:93-196, 1974.

[10] Pion Mass Collaboration. Measurement Of The Pion Mass. PSI experiment proposal R-97.02, 1997. 
